\title{
Fatigue Life Calculation Method and Experimental Study of the Multiple Corrugated Diaphragm Coupling
}

\author{
Angang Cao $\mathbb{D}^{1}{ }^{1}$ Chunhua Ding, ${ }^{2}$ Wei Li, ${ }^{1}$ and Zhiyong Zhang ${ }^{1}$ \\ ${ }^{1}$ School of Mechanical Engineering, Zhengzhou University of Science and Technology, Zhengzhou 450064, China \\ ${ }^{2}$ The 703 Research Institute of CSSC, Harbin 150078, China \\ Correspondence should be addressed to Angang Cao; caoangang88@163.com
}

Received 16 August 2021; Revised 18 October 2021; Accepted 15 November 2021; Published 27 November 2021

Academic Editor: Nur Izzi Md. Yusoff

Copyright (c) 2021 Angang Cao et al. This is an open access article distributed under the Creative Commons Attribution License, which permits unrestricted use, distribution, and reproduction in any medium, provided the original work is properly cited.

The multiple corrugated diaphragm (MCD) coupling is a new flexible coupling developed based on the diaphragm and diaphragm disc coupling. Compared to traditional couplings, the MCD coupling has the advantages of high torque diameter, high torque weight, and high compensation capability. It is more suitable for high power speed and high power density working conditions. The MCD coupling is subjected to axial, angular, torque, and centrifugal force loads. The fatigue failure caused by alternating stress is the primary failure mode of the coupling. The fatigue life of the MCD coupling cannot be accurately calculated because of the complexity of the force in operation. Some theoretical simplifications can only obtain the approximate result. In this paper, a parameterized finite element model of the MCDs is established. A method for calculating the fatigue safety factor of the MCD coupling is proposed based on a modified Goodman curve to know the design of the MCD coupling. The feasibility of this method is verified by the fatigue life test of the coupling.

\section{Introduction}

The multiple corrugated diaphragm (MCD) coupling is a new flexible coupling developed based on the diaphragm and diaphragm disc coupling. Its core component is the high strength and high toughness stainless steel diaphragm disk group. Multiple separated diaphragm discs in the disc group have an internal fault protection function, making the coupling have greater flexibility and obtain greater torque transmission capacity without increasing the diameter of the coupling. In power transfer and deformation compensation, the parallel corrugated diaphragm increases the profile by expanding the wave. Therefore, compared with the traditional coupling, the MCD coupling has the advantages of high torque diameter ratio, high torque weight ratio, and large compensation capacity, which is more suitable for increased power and speed conditions [1-4].

Previous researchers conducted several studies on the coupling applied in different fields. Nagesh et al. conducted fatigue analysis on metal diaphragm couplings of various materials through test data obtained from the S-N curve.
They analyzed the influence of angle offset on the fatigue life of metal diaphragm couplings [5]. Verucchi et al. carried out an experimental study on the structure of the diaphragm coupling of the turbine. They proved that the diaphragm structure with shot-peening treatment could improve the fatigue life of the design by at least 10\% [6]. Li et al. introduced the coupling and other coupling mechanisms in their work [7]. Herbstritt et al. introduced an economical coupling [8]. Duong and Kazerounian designed an improved diaphragm coupling for aerospace applications [9]. Daniel discussed the virtual entity method of creating elastic coupling with computer-aided technology and completed the modeling design of the flexible coupling model [10]. Dobre et al. carried out finite element analysis on the elastic coupling and demonstrated the influence of axial deviation on the diaphragm coupling in the process of transmitting torque; that is, the maximum equivalent stress increases with the increase of axial deviation [11]. Wang and Chang studied the axial vibration characteristics of the coupling and demonstrated the shock absorption characteristics of the coupling as an elastic coupling mechanism [12]. Farias et al. 
discussed the metal components of the coupling and summarized the categories and suitable types of various metal components in the coupling [13]. Lun et al. analyzed the strength and vibration characteristics of the diaphragm coupling. They showed the influence of vibration characteristics of the diaphragm coupling on the strength under the working state [14]. Mancuso introduced several couplings for gas turbines and mentioned that the multiple corrugated diaphragm couplings are more suitable for gas turbines. Still, the design and calculation methods of corrugated film couplings were not introduced [15]. Li studied the corrugated diaphragm's structural strength, fatigue life, and vibration characteristics by establishing the parametric finite element model of the corrugated diaphragm [16]. Yang et al. used the MSC Patran module in finite element analysis software to check the nonlinear strength of the diaphragm coupling [17]. Li and Li analyzed the dynamic characteristics and vibration mechanism of parallel misalignment and angular misalignment middle rotors by building a multispan rotor-bearing system testbed [18]. Feng et al. established the model of trunnion joint to study the influence of rotating speed and the ratio of inner radius to the outer radius on the axial stiffness, which provides a reference for designers to choose the proper ratio of inner radius to the outer radius of the rotating circular plate [19].

According to the existing references, many research institutions on diaphragm couplings, membrane disc couplings, and laminated couplings have been more in depth. Many scholars carried out extensive research from the theoretical calculation and finite element analysis to the stress, stiffness, and fatigue life test. The diaphragm coupling, membrane disc coupling, laminated coupling stress analysis, fatigue life analysis, design method, processing technology, test method, and installation application have an emotional experience. However, research studies on the MCD coupling are relatively few. The research on the fatigue stress and fatigue life of the MCD coupling is even less. For the design of the coupling, fatigue life is a problem that must be considered and paid attention to. Therefore, it is necessary to study the fatigue stress and fatigue life of the MCDs to promote the application and development of the MCD coupling.

In this paper, the finite element model of the MCDs is established by Ansys finite element analysis software. A method for calculating the fatigue safety factor of the MCD coupling is presented based on the third strength theory and modified Goodman curve. Then, the fatigue life test method of the MCD coupling is designed. The axial fatigue life test and angular fatigue life test are carried out, which verify the feasibility of the calculation method of the fatigue safety factor of the MCDs to a certain extent.

\section{Geometric Parameters and Working Parameters of the MCDs}

The shape of the MCDs is shown in Figure 1. And the specific geometric parameters of the MCDs in this paper are shown in Table 1. The influence of the bolt hole and spline was ignored in the subsequent analysis [4]. The load parameters of the MCDs in this paper are shown in Table 2.

\section{Fatigue Safety Factor of the MCDs}

Disk couplings require very high processing technology and unique materials mainly because they adapt to moderate flexibility, so fatigue strength is their main performance index. To determine fatigue strength, the method proposed by NB Rothfuss is to draw the average composite stress and alternating composite stress on the fatigue diagram of equal life and set $10^{7}$ cycle lifelines as the fatigue limit parameter with infinite life limit. For fatigue diagrams, three average stress correction methods have been invented to avoid the trouble of fatigue tests under different average stresses, namely, Goodman method, Gerber method, and Soderberg method. The three modified fatigue diagrams are shown in Figure 2. Considering the dispersion of fatigue data, the Gerber parabola is not safe, and the calculation is complicated. The Soderberg line can make safe predictions, and in many cases, it seems overly safe. Although the Goodman line is not $100 \%$ safe, it is much safer than the Gerber parabola, and it is easy to calculate, so it is widely used in the fatigue design of mechanical parts. In this paper, the Goodman curve is simplified into a straight line, and the modified Goodman curve is used as the infinite lifeline to calculate the fatigue safety factor [20].

According to the Ansys help file, we can see that Plane42 is defined by four nodes having two degrees of freedom at each node: translations in the nodal $x$ - and $y$-directions. Plane25 is defined by four nodes having three degrees of freedom per node: translations in the nodal $x$-, $y$-, and $z$-direction. Plane 25 is a generalization of the axisymmetric version of Plane42. Obviously, for the MCDs, only the $x$ - and $y$-direction is not enough, and Plane25 is more suitable for the analysis of MCDs. In addition, the FE model is characterized by using a simple two-dimensional model to analyze three-dimensional forces. Therefore, this paper adopts the Plane25 element for FE modeling.

In Ansys software, APDL (Ansys Parametric Design Language) was used for parametric modeling, and Plane25 element was used to mesh the membrane disc with quadrilateral ring elements $[1,2,4]$. The finite element model of the MCDs was obtained [21-26]. By applying an axial load, angular load, centrifugal force load, and torque load to the finite element model of the MCDs, the radial stress, tangential stress, and torsional shear stress of the MCDs under different loads can be obtained [27-29].

The stress generated by the axial load and the centrifugal load of the MCDs is in the same plane, so the two stresses can be combined linearly:

$$
\begin{aligned}
& \sigma_{r}=\sigma_{r x}+\sigma_{r l}, \\
& \sigma_{t}=\sigma_{t x}+\sigma_{t l} .
\end{aligned}
$$

In the formula, $\sigma_{r}$ is the synthetic radial stress; $\sigma_{t}$ is the synthetic tangential stress; $\sigma_{r x}$ is the radial stress due to axial load; $\sigma_{t x}$ is the tangential stress due to axial load; $\sigma_{r l}$ is the radial stress generated under centrifugal force loading; $\sigma_{t l}$ is the tangential stress generated under centrifugal force loading.

Therefore, the maximum and minimum principal stresses of the static stress of the MCDs are 


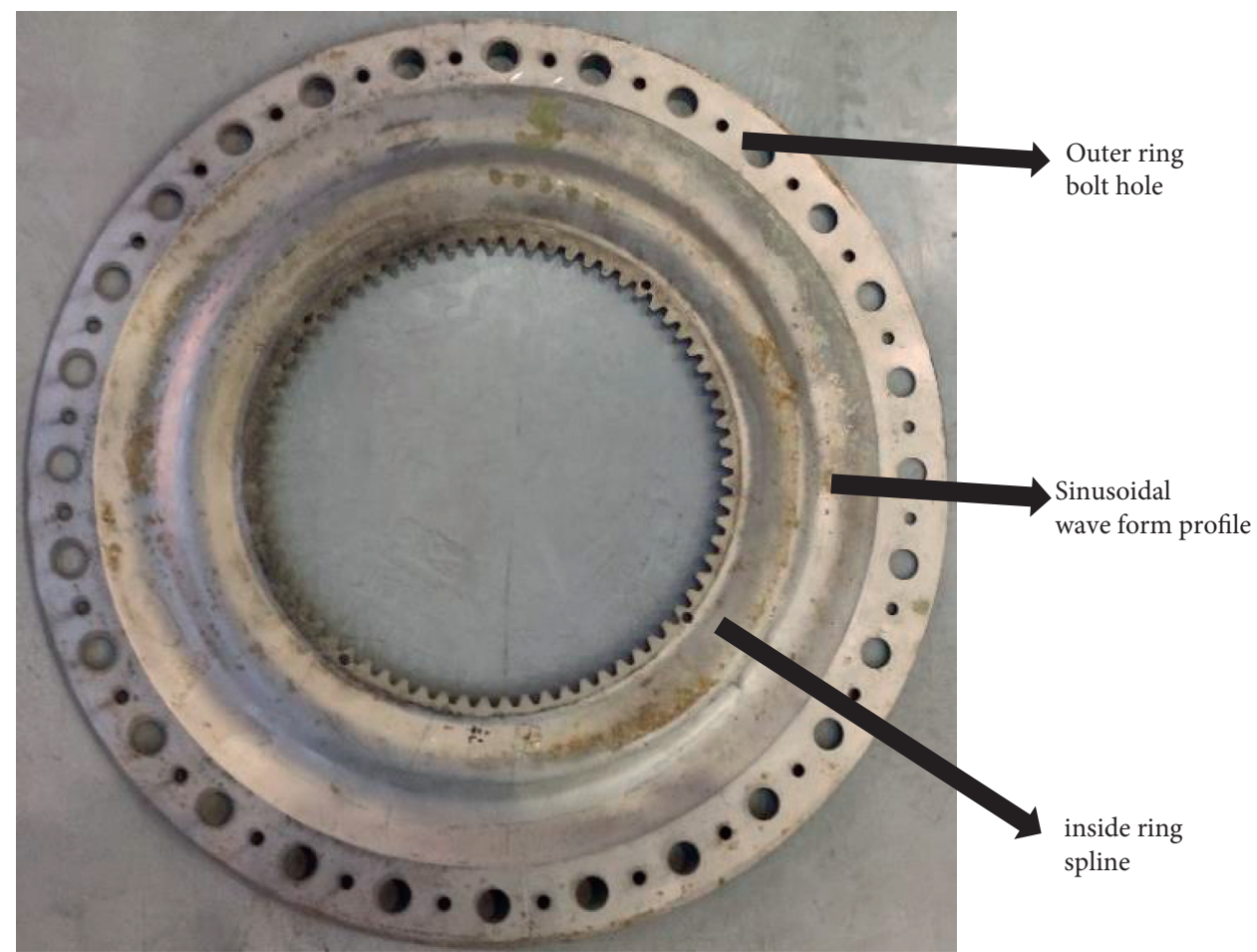

Figure 1: The shape of the MCDs.

TABle 1: Geometric parameters of the MCDs.

\begin{tabular}{lccc}
\hline Serial number & Name of the parameter & Symbol (unit) & Value \\
\hline 1 & The inner radius of the profile & $a(\mathrm{~mm})$ & 104 \\
2 & The outside radius of the profile & $b(\mathrm{~mm})$ & 162 \\
3 & The thickness of the profile & $t(\mathrm{~mm})$ & 0.5 \\
4 & The amplitude of the profile & $c(\mathrm{~mm})$ & 3.5 \\
5 & The thickness of the hub & $h_{1}(\mathrm{~mm})$ & 1.2 \\
6 & The thickness of the flange & $h_{2}(\mathrm{~mm})$ & 1.2 \\
7 & The radius of the transition fillet & $r_{2}(\mathrm{~mm})$ & 2 \\
8 & The radius of the inner circle & $r_{0}(\mathrm{~mm})$ & 89 \\
9 & The radius of the outer circle & $r_{1}(\mathrm{~mm})$ & 182 \\
10 & The number of diaphragms in an assembly & $m(\mathrm{piece})$ & 15 \\
\hline
\end{tabular}

TABLE 2: Geometric parameters of the MCDs.

\begin{tabular}{lccc}
\hline Axial load $(\mathrm{mm})$ & Angle load $\left(^{\circ}\right)$ & Torque load $(\mathrm{N} \cdot \mathrm{m})$ & Centrifugal load $(\mathrm{r} / \mathrm{min})$ \\
\hline 2.5 & 0.25 & 55148 & 5200 \\
\hline
\end{tabular}

$$
\begin{gathered}
\sigma_{\text {staticmax }}=\frac{\sigma_{r}+\sigma_{t}}{2}+\sqrt{\frac{\left(\sigma_{r}-\sigma_{t}\right)^{2}}{4}+\tau^{2}}, \\
\sigma_{\text {staticmin }}=\frac{\sigma_{r}+\sigma_{t}}{2}-\sqrt{\frac{\left(\sigma_{r}-\sigma_{t}\right)^{2}}{4}+\tau^{2}} .
\end{gathered}
$$
load.

In the formula, $\tau$ is the shear stress generated by torque

The dynamic stress of the MCDs is the alternating stress caused by angular deformation. The MCDs change once per rotation, which also belongs to the plane stress state.
Therefore, the maximum and minimum principal stresses of the dynamic stress are

$$
\begin{gathered}
\sigma_{\text {dynamicmax }}=\sigma_{r f}, \\
\sigma_{\text {dynamicmin }}=\sigma_{t f} .
\end{gathered}
$$

In the formula, $\sigma_{r \phi}$ is the radial stress under angular load, and $\sigma_{t \phi}$ is the tangential stress under angular load.

According to the third strength theory, the static and dynamic working stresses of the MCD can be obtained as follows [30].

When $\sigma_{\max } \sigma_{\min }<0$, the working stress is 


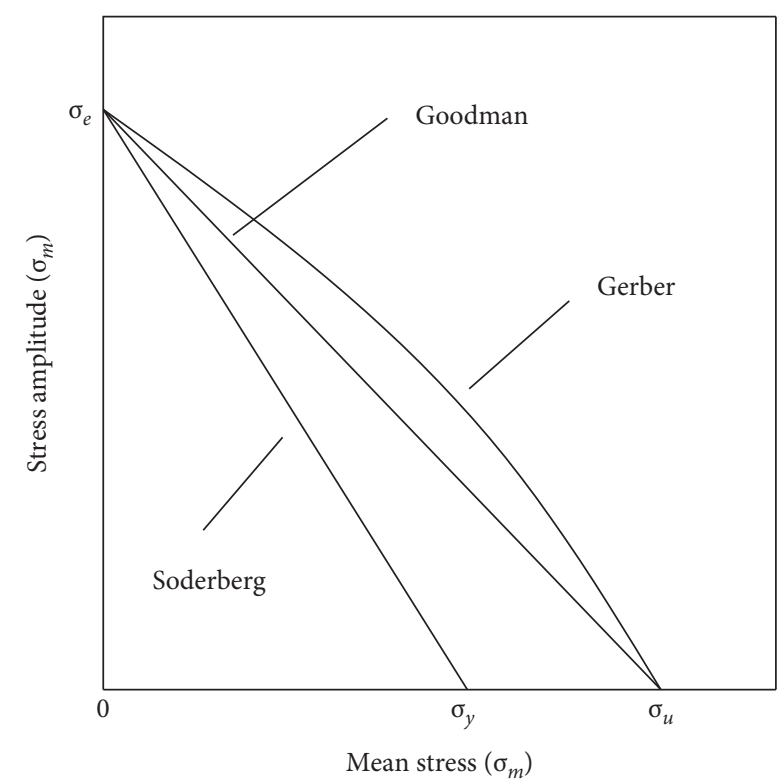

FIgURE 2: Three average stress correction methods.

$$
\sigma=\left|\sigma_{\max }\right|+\left|\sigma_{\min }\right|
$$

When $\sigma_{\max } \sigma_{\min }>0$, the working stress is

$$
\sigma=\max \left\{\left|\sigma_{\max }\right|,\left|\sigma_{\min }\right|\right\} \text {. }
$$

If only symmetrical cyclic alternating stress is assumed, the working stress is required to be $\sigma_{\text {dynamic }} \leq\left[\sigma_{\text {dynamic }}\right]$. When only static stress is applied, the operational stress is needed to be $\sigma_{\text {static }} \leq\left[\sigma_{\text {static }}\right]$. In the formula, $\left[\sigma_{\text {dynamic }}\right]$ is the allowable dynamic stress, and $\left[\sigma_{\text {static }}\right]$ is the allowable static stress. In the general case, under this cyclic characteristic, the line between the composite stress $P$ of static and dynamic stresses and the origin $O$ and the intersection point of the modified Goodman curve is $M$. It is safe when the ratio of $A$ to $B$ is more significant than one. The modified Goodman curve is assumed to be a straight line for simplicity, as shown in Figure 3.

Then, the safety factor of the MCD coupling is

$$
n=\frac{\overline{O M}}{\overline{O P}} .
$$

According to the above derivation, fatigue safety factors at each position of the profile line of the MCD coupling can be obtained by using Ansys software, as shown in Figure 4. The safety factor at each position of the MCD profile line is greater than 1 , and the minimum value is 1.34 , which meets the design requirements.

\section{Fatigue Life Test of the MCD Coupling}

During the coupling operation, the external axial or angular excitation will inevitably lead to the accelerated destruction of the flexible components, which will reduce the life of the coupling. Under the rated compensation capacity and torque, the stress level on the flexible components of the coupling is far below the material's ultimate strength, and

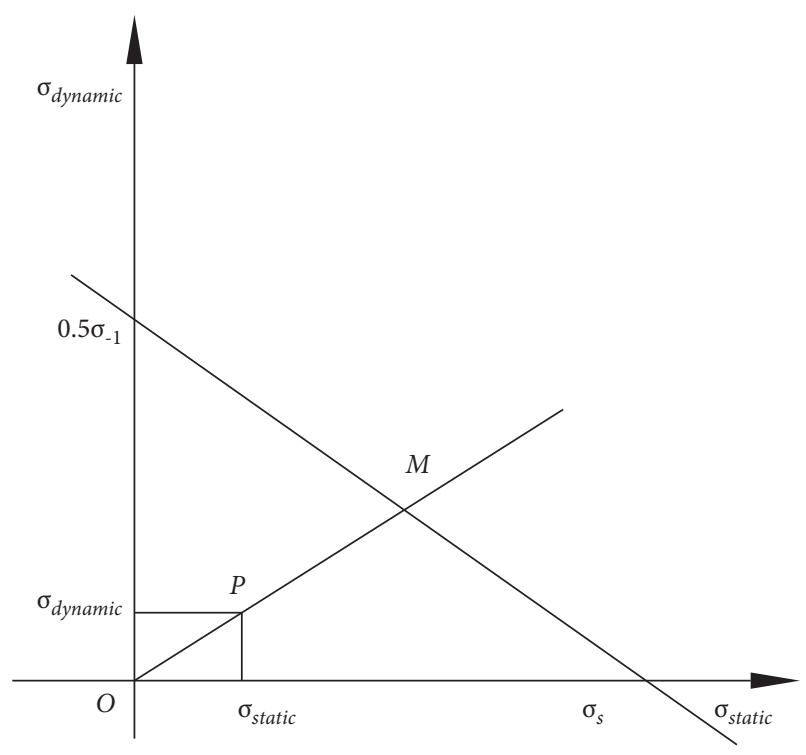

Figure 3: Goodman curve.

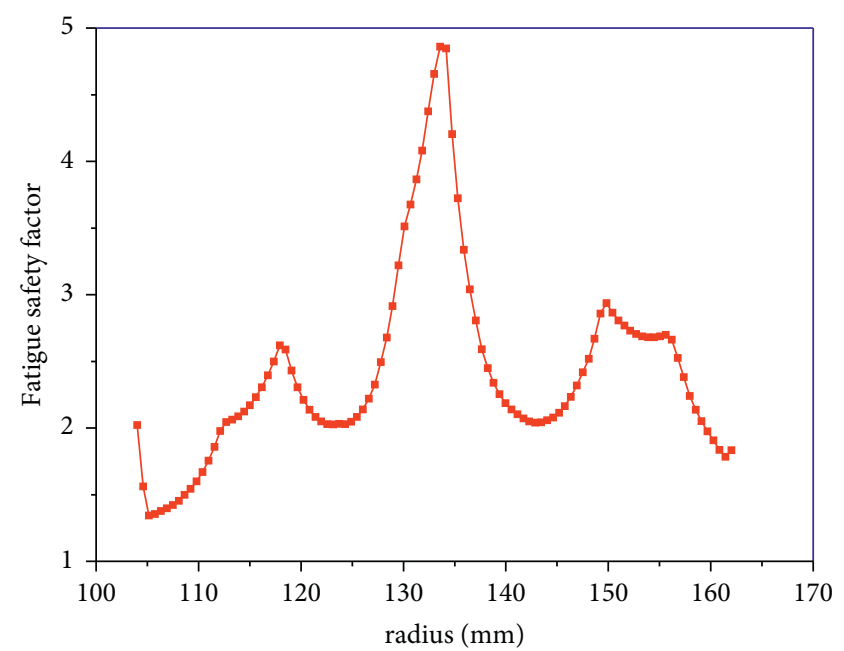

Figure 4: The fatigue safety factor of the MCD coupling.

the fatigue stress is the direction that the coupling design needs to focus. The fatigue failure caused by alternating stress is the primary failure mode of the coupling. In this paper, through the fatigue life test of the coupling, the feasibility of calculating the fatigue safety factor of the MCD coupling is verified.

4.1. Accelerated Fatigue Test Principles. The coupling is generally designed according to the infinite life or the same life as the unit, but the life design is usually calculated by 20 years. Therefore, the accelerated equivalent fatigue test must be carried out because the cycle time of regular fatigue tests is too large and time-consuming. According to the identical fatigue test method, the equivalent fatigue test of the coupling can be carried out by increasing the excitation frequency and vibration amplitude. According to the sinusoidal 


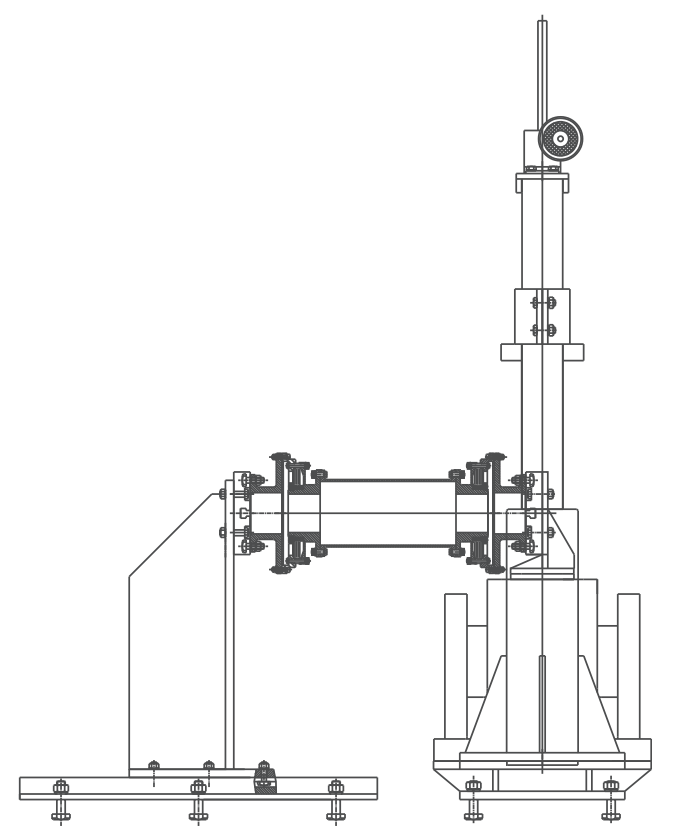

(a)

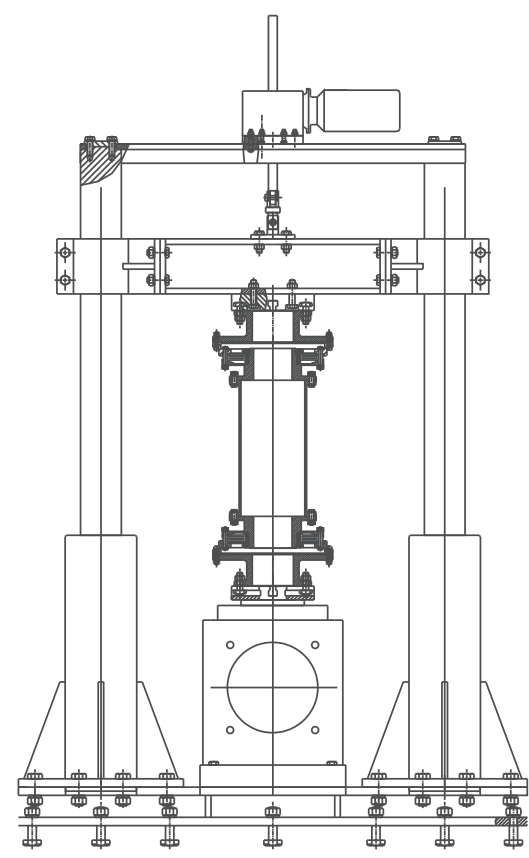

(b)

Figure 5: Coupling vibration test bench. (a) Angular vibration test. (b) Axial vibration test.

vibration fatigue equivalent test method, there is a relationship between the test time before and after the equivalent test and the excitation amplitude as follows:

$$
\frac{t_{1}}{t_{2}}=\left(\frac{\ddot{x}_{2}}{\ddot{x}_{1}}\right)^{k}=\left(\frac{s_{2}}{s_{1}} \times \frac{t_{1}^{2}}{t_{2}^{2}}\right)^{k}
$$

In the formula, $t_{1}$ and $t_{2}$ are the time before and after the equivalent test, and $\ddot{x}_{1}$ and $\ddot{x}_{2}$ are the acceleration magnitudes before and after the equivalent test. $K$ value depends on material damping characteristics, geometric shape, loading form, and temperature environmental conditions; $K=6$ is recommended in similar trials in the United States; in China, $K$ value is determined to be between 5 and 7 through many tests. This paper prefers to be conservative, $K=5$.

Because of the limitation of the flexible coupling compensation performance test of vibration amplitude adjustment, damaged coupling compensation is too significant and too much. To accelerate the fatigue test, this test will be set to $10 \mathrm{~mm}$ axial vibration amplitude, and the angular amplitude is set as $5.74 \mathrm{~mm}$. In this case, the axial vibration amplitude is two times of the rated axial compensation of the coupling. Also, the middle length of the coupling is $658 \mathrm{~mm}$, so the angular amplitude is about 2 times of the rated angular compensation.

For the axial vibration, the excitation comes from the axial vibration of the main engine. The primary engine excitation frequency is taken at 20 , and the unit works 24 hours a day; then, the coupling fatigue alternating times within 20 years are

$$
N_{1}=t_{\text {total }} \times f=20 \times 365 \times 24 \times 3600 \times 20=1.26 \times 10^{10} \text {. }
$$

Set the excitation frequency of the equivalent test as $35 \mathrm{~Hz}$ and the excitation amplitude as $10 \mathrm{~mm}$; then, according to equation (7), the number of cycles in the identical test is $1.46 \times 10^{6}$ times, about 11.6 hours.

For the angular vibration, the excitation comes from the combined action of angular deviation and circular rotation. The coupling speed is $5200 \mathrm{r} / \mathrm{min}$; the unit works 24 hours a day; then, the coupling fatigue alternating times in 20 years are

$$
N_{2}=t_{\text {total }} \times f=20 \times 365 \times 24 \times 3600 \times \frac{5200}{60}=5.46 \times 10^{10} .
$$

Set the excitation frequency of the equivalent test as $150 \mathrm{~Hz}$ and the excitation amplitude as $5.74 \mathrm{~mm}$; then, according to equation (7), the number of cycles in the identical test is $7.09 \times 10^{6}$ times, about 13.1 hours.

4.2. Accelerated Fatigue Life Test Schemes. Fatigue damage of the flexible coupling refers to that, in the use process, under the condition of repeated stress, the performance of flexible components will be reduced or damaged so that they cannot continue to use.

In this paper, the accelerated fatigue test of the MCD coupling was carried out on the vibration testbed of the coupling (as shown in Figure 5). The influence of fatigue deformation was judged by the axial stiffness changed before and after the test.

The test scheme is as follows:

(1) The axial stiffness of two groups of the MCD assemblies (numbered $1 \#$ and 2\#, respectively) in the 


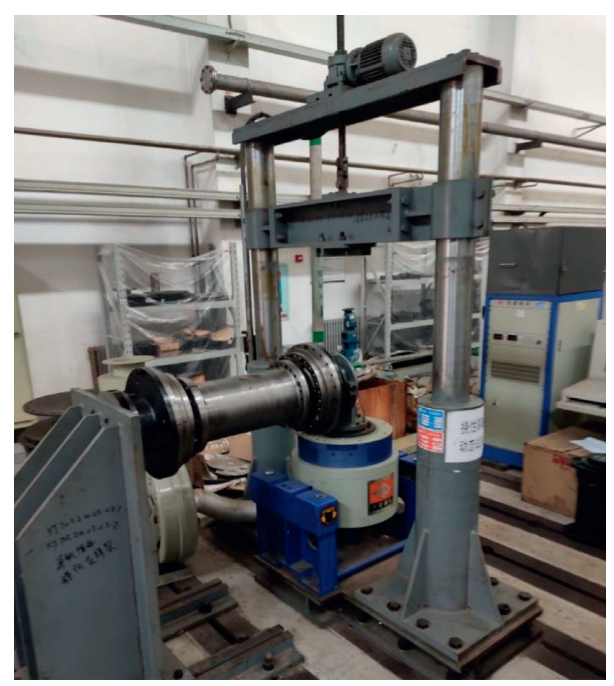

Figure 6: Angular vibration test of the MCD coupling.

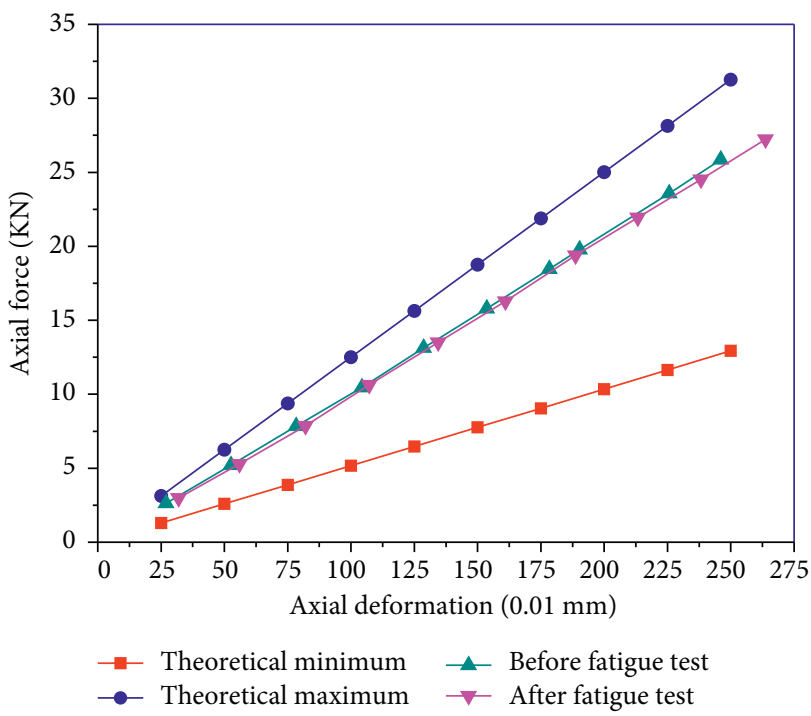

(a)

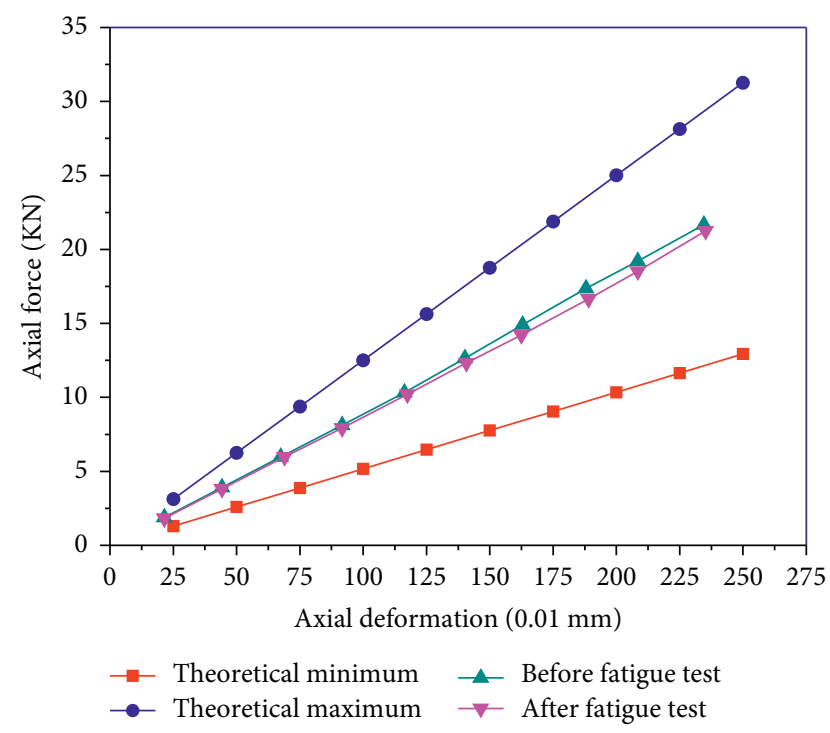

(b)

Figure 7: Axial stiffness curve of the MCD assemblies before and after the axial fatigue life test. (a) The MCD assemblies of 1\#. (b) The MCD assemblies of $2 \#$.

prototype was measured, and the results were recorded.

(2) The prototype of the MCD coupling was installed in the axial vibration test position of the coupling vibration testbed. The vibration frequency of the testbed was adjusted to $35 \mathrm{~Hz}$, the vibration amplitude was adjusted to $10 \mathrm{~mm}$, and the 12-hour accelerated fatigue test was carried out.

(3) Remove the prototype, check whether there is any damage in the appearance of the MCDs, and then measure the axial stiffness of the two groups of the MCD assemblies and record the results.

(4) Install the prototype of the MCD coupling in the angular vibration test position of the coupling vibration test bench (as shown in Figure 6), adjust the vibration frequency of the test bench to $150 \mathrm{~Hz}$ and the vibration amplitude to $5.74 \mathrm{~mm}$, and conduct a 14-hour accelerated fatigue test.

(5) Remove the prototype, check whether there is any damage in the appearance of the MCDs, and then 


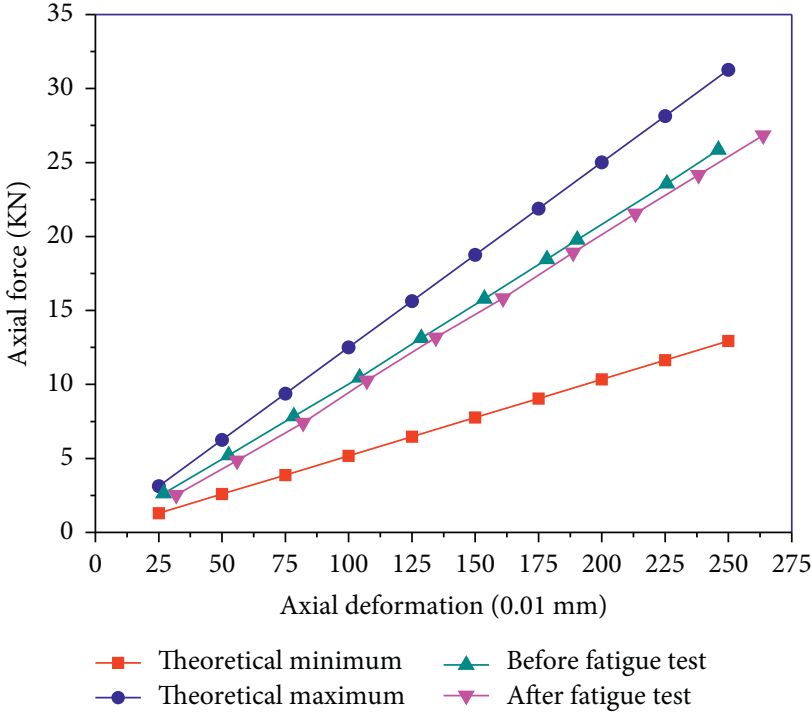

(a)

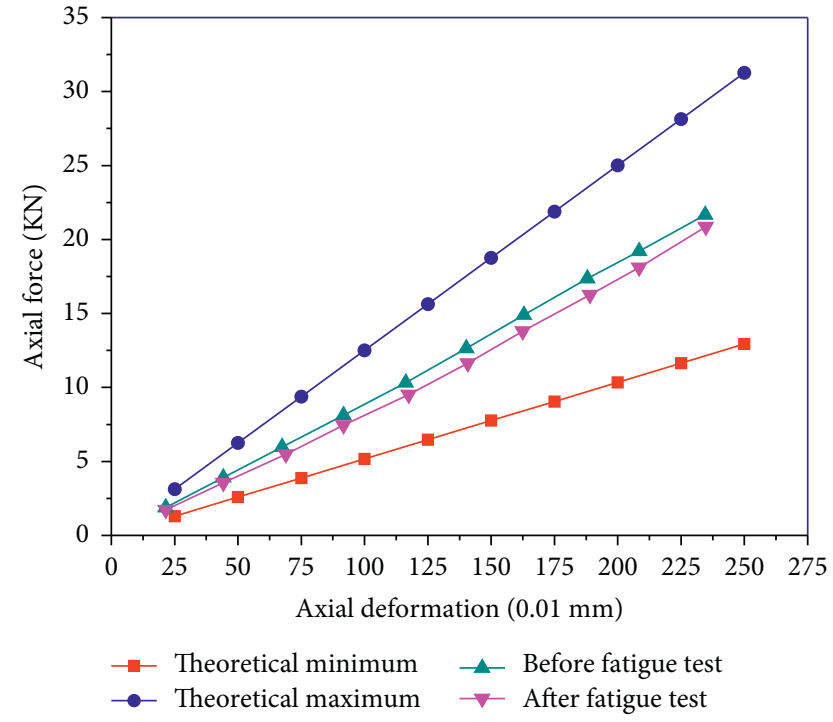

(b)

Figure 8: Axial stiffness curve of the MCD assemblies before and after the angular fatigue life test. (a) The MCD assemblies of $1 \#$. (b) The MCD assemblies of 2\#.

TABLE 3: Stiffness value of the MCD assemblies before and after the fatigue test.

\begin{tabular}{lccccc}
\hline $\begin{array}{l}\text { Part } \\
\text { number }\end{array}$ & $\begin{array}{c}\text { Axial stiffness } \\
\text { before test }(\mathrm{kN} / \\
\mathrm{mm})\end{array}$ & $\begin{array}{c}\text { Axial stiffness after the } \\
\text { axial fatigue test }(\mathrm{kN} / \\
\mathrm{mm})\end{array}$ & $\begin{array}{c}\text { Relative to the change } \\
\text { before the experiment } \\
(\%)\end{array}$ & $\begin{array}{c}\text { Axial stiffness after the } \\
\text { angular fatigue test } \\
\text { mm })\end{array}$ & $\begin{array}{c}\text { Relative to the change } \\
\text { before the experiment } \\
(\%)\end{array}$ \\
\hline 1 & 10.198 & 9.948 & 2.46 & 9.522 & 6.63 \\
2 & 9.01 & 8.73 & 3.10 & 8.323 & 7.61 \\
\hline
\end{tabular}

measure the axial stiffness of the two groups of the MCD assemblies and record the results.

\section{Results and Discussion}

After inspection, no damage was found in the appearance of the two MCD assemblies after the axial fatigue life test. After sorting, the axial stiffness test data of the MCD assemblies before and after the axial fatigue life test are shown in Figure 7.

After inspection, no damage was found in the appearance of the two MCD assemblies after the angular fatigue life test. After sorting, the axial stiffness test data of the MCD assemblies before and after the angular fatigue life test are shown in Figure 8.

The average axial stiffness of the two groups of MCD assemblies before and after the fatigue test is shown in Table 3.

After the fatigue test, no damage was found in the two MCD assemblies. It can be seen from the stiffness curves before and after the fatigue life test of the MCDs in Figures 7 and 8 and the stiffness values of MCD assemblies before and after the fatigue test in Table 3 that, after the axial fatigue test, the axial stiffness of 1 \# MCD assemblies decreased by $2.46 \%$, and the axial stiffness of $2 \#$ MCD assemblies decreased by $3.10 \%$; after the angular fatigue test, the axial stiffness of $1 \#$ MCD assemblies and 2\# MCD assemblies decreased by
$4.17 \%$ and $4.51 \%$, respectively, compared with that before the fatigue test. After the two fatigue tests, the stiffness of $1 \#$ MCD assemblies decreased by $6.63 \%$, and the axial stiffness of 2\# MCD assemblies decreased by $7.61 \%$ compared with that before the tests. The results show that the axial stiffness of the MCDs has little change after the fatigue test, and the performance of the MCD coupling in the fatigue life can meet the design requirements.

\section{Conclusions}

In this paper, the parameterized finite element model of the MCDs was established by Ansys software, and the following results were obtained:

(1) A method for calculating the fatigue safety factor of the MCDs is presented. Based on this, the MCDs can be optimized to improve the performance of the MCD coupling.

(2) The axial and angular accelerated fatigue life tests of the MCD coupling are carried out using the couplings' vibration testbed. After the fatigue life test, the axial stiffness of the MCD assemblies is reduced compared with that before the test. However, it is still within the design range, which proves that the performance of the MCDs designed in this paper meets the design requirements during the fatigue life 
period and verifies the feasibility of the calculation method of the fatigue safety factor of the MCD coupling proposed in this paper.

\section{Data Availability}

The data used to support the findings of this study are available from the corresponding author upon request.

\section{Conflicts of Interest}

Angang Cao received a $\mathrm{PhD}$ degree from Harbin Shipbuilding Boiler Turbine Research Institute, Harbin, China, in 2020. Now, he is an Associate Professor at the Zhengzhou University of Science and Technology. His current research interests include mechanical transmission, machinery dynamics, and mechanical structure. Chunhua Ding received a master's degree from Harbin Shipbuilding Boiler Turbine Research Institute, Harbin, China, in 2011. Now, he is a professor of engineering in the CSIC No. 703 Research Institute. His current research interests include mechanical transmission, machinery dynamics, and mechanical structure. Wei Li received a master's degree from Xi'an University of Technology, Xi'an, China, in 2010. Now, he is an Associate Professor at the Zhengzhou University of Science and Technology. His current research interests include mechanical transmission, machinery dynamics, and mechanical structure. Zhiyong Zhang received a master's degree from Zhengzhou University, Zhengzhou, China, in 2014. Now, he is a lecturer at the Zhengzhou University of Science and Technology. His current research interests include mechanical transmission, machinery dynamics, and mechanical structure.

\section{Acknowledgments}

This work was supported by the Key Scientific Research Project of Colleges and Universities of Henan Provincial Department of Education (nos. 20B460016, 21B460020, and 22B460027).

\section{References}

[1] A. Cao, C. Ding, and W. Xu, "Study on optimization of waveform diaphragm molded lines," Journal of Engineering for Thermal Energy and Power, vol. 34, no. 1, pp. 120-124, 2019, (in Chinese).

[2] A. Cao, S. Chang, C. Ding, and S. Li, "Design and optimization of transition fillet for waveform diaphragm," Ship Science and Technology, vol. 40, no. 4, pp. 139-142, 2018, (in Chinese).

[3] A. Cao, L. Xing, and X. Liu, "Torsional stiffness of waveform diaphragm with sinusoidal molded lines on the basis of membrane theory," Journal of Engineering for Thermal Energy and Power, vol. 35, no. 8, pp. 158-164, 2020, (in Chinese).

[4] A. Cao, L. Xing, X. Zheng, and Y. Zhu, "Analytical and experimental study on the axial stiffness of multiple corrugated diaphragm coupling based on the corrugated circular plate theory," Proceedings of the Institution of Mechanical Engineers - Part C: Journal of Mechanical Engineering Science, Article ID 095440622110145, 2021.
[5] S. Nagesh, A. M. Junaid Basha, and T. D. Singh, "Dynamic performance analysis of high speed flexible coupling of gas turbine engine transmission system," Journal of Mechanical Science and Technology, vol. 29, no. 1, pp. 173-179, 2015.

[6] C. Verucchi, J. Bossio, G. Bossio, and G. Acosta, "Misalignment detection in induction motors with flexible coupling by means of estimated torque analysis and MCSA," Mechanical Systems and Signal Processing, vol. 80, pp. 570-581, 2016.

[7] J. J. Li, E. C. Yang, W. J. Liu, and Y. Li, "Coupling modeling and analysis of a wind energy converter," Advances in $\mathrm{Me}$ chanical Engineering, vol. 8, no. 6, 2016.

[8] W. R. Herbstritt, D. J. Konopka, and T. L. Slagle, "Introduction of an economical diaphragm coupling for mid-range applications," in Proceedings of the 2000 ASME design engingeering technical conference, p. 13, Baltimore, MD, USA, September 2000.

[9] L. Duong and K. Kazerounian, "Design improvement of the mechanical coupling diaphragms for aerospace applications\#," Mechanics Based Design of Structures and Machines, vol. 35, no. 4, pp. 467-479, 2007.

[10] D. Daniel, "Computer aided design of elastic coupling with metallic membranes using solid modeling," JIDEG, vol. 3, no. 4, pp. 21-24, 2008.

[11] D. Dobre, I. Simion, V. G. Adir, G. M. Adir, and N. Pascu, "Finite element analysis of the flexible coupling with metallic membranes," Annals of DAAAM \& Proceedings, vol. 1, pp. 421-423, 2010.

[12] X. F. Wang and D. G. Chang, "Kinematic and dynamic analyses of tripod sliding universal joints," Journal of Mechanical Design, vol. 131, no. 6, Article ID 061011, 2009.

[13] R. C. Farias, J. E. Cohen, C. Jutten, and P. Comon, "Joint decompositions with flexible couplings," in Proceedings of the Latent Variable Analysis and Signal Separation, Liberec, Czech Republic, August 2015.

[14] L. Lun, C. Dengqing, and W. Jin, "Rigid flexible coupling dynamic modeling and vibration control for flexible spacecraft based on its global analytical modes," Science China (Technological Sciences), vol. 62, no. 4, pp. 92-102, 2019.

[15] J. R. Mancuso, "Flexible couplings for gas turbine applications," in Proceedings of the ASME 1989 International Gas Turbine and Aeroengine Congress and Exposition, Toronto, Canada, June 1989.

[16] X. Li, "Structure analysis and optimization of marine corrugated diaphragm coupling," Master's Thesis (in Chinese), Harbin Engineering University, Harbin, China, 2018.

[17] L. Yang, X. Xu, H. Qiao, and C. Zeng, "Finite element analysis of elastic membrane coupling based on MSC. Patran," Machinery, vol. 42, no. 1, pp. 50-52, 2015, (in Chinese).

[18] M. Li and Z. Li, "Theoretical and experimental study on dynamics of rotor-bearing system with the faults of coupling misalignment," Journal of Vibration, Measurement \& Diagnosis, vol. 35, no. 2, pp. 345-351, 2015, (in Chinese).

[19] S. Feng, H. Geng, B. Zhang, L. Yang, and L. Yu, "Axial stiffness of a rotating trunnion joint," Proceedings of the Institution of Mechanical Engineers - Part C: Journal of Mechanical Engineering Science, vol. 229, no. 16, pp. 2846-2853, 2014.

[20] C. Yue, S. Guo, and M. Li, "Ansys fluent based modeling and hydrodynamic analysis for a spherical under water robot," in Proceedings of the IEEE International Conference on Mechatronics \& Automation, IEEE, Takamatsu, Japan, August 2013.

[21] ANSYS Inc, ANSYS Modeling and Meshing Guide, ANSYS. Inc, Canonsburg, PA, USA, 2004.

[22] K. Bao, Q. Zhang, Y. Liu, and D. Jin, "Fatigue life of the welding seam of a tracked vehicle body structure evaluated 
using the structural stress method," Engineering Failure Analysis, vol. 120, 2021 (in Chinese).

[23] R. Unarine, D. Desai, S. Glen, and J. Tamba, "Significance of residual stresses in fatigue life prediction of micro gas turbine blades," Engineering Failure Analysis, vol. 120, 2021.

[24] W. Jiang, X. Xie, T. Wang et al., "Fatigue life prediction of 316L stainless steel weld joint including the role of residual stress and its evolution: experimental and modeling," International Journal of Fatigue, vol. 143, 2021.

[25] H. Feng, Y. Wang, and X. Jiang, "A maximum likelihood method for estimating probabilistic strain amplitude-fatigue life curves," Acta Mechanica Solida Sinica, vol. 31, no. 1, pp. 80-93, 2018.

[26] S. R. Prasad and A. S. Sekhar, "Life estimation of shafts using vibration based fatigue analysis," Journal of Mechanical Science and Technology, vol. 32, no. 9, pp. 4071-4078, 2018.

[27] Q. B. Liu, W. K. Shi, and Z. Y. Chen, "Rubber fatigue life prediction using a random forest method and nonlinear cumulative fatigue damage model," Journal of Applied Polymer Science, vol. 137, no. 14, 2020.

[28] X. H. Deng, "Analysis and prediction of gear fatigue life," in Proceedings of the 2018 4th International Conference on Environmental Science and Material Application, Xi'an, China, December 2018.

[29] P. M. Wavish, I. R. Mccoll, and S. B. Leen, "Design of a multiaxial test specimen for fretting fatigue in splined couplings," Advances in Experimental Mechanics IV, vol. 8, pp. 267-272, 2005.

[30] X. F. Yang, X. U. Wei, and G. P. Guo, "Experimental study of vibration fatigue of coupling diaphragm based on nonlinear vibration," Journal of Experimental Mechanics, vol. 31, no. 6, pp. 763-768, 2016. 